\title{
Les erreurs de mesure en céphalométrie 2D
}

\author{
Jérôme BOURRIAU, Guillaume BIDANGE, Jean-Michel FOUCART*
}

CHU Hôtel Dieu (AP-HP), 1 place du Parvis Notre-Dame, 75181 Paris Cedex 4, France

MOTS CLÉS :

Céphalométrie /

Erreur /

Repère /

Mesure

KEYWORDS:

Cephalometry /

Error /

Landmark/

Measurement
RÉSUMÉ - L'utilisation de la téléradiographie de profil (norma latéralis) à des fins cliniques est très étroitement liée à la précision d'identification et de localisation des différents " points » requis lors de l'élaboration des différentes analyses céphalométriques. L'objet de cette étude est d'analyser l'influence de deux paramètres que sont la distance foyer-objet et le type de récepteur, sur la précision de localisation des 19 points céphalométriques les plus usités puis d'analyser la répercussion des erreurs de localisation sur les mesures céphalométriques. Un groupe de 53 orthodontistes a été aléatoirement sélectionné ; tous ont identifié 19 points céphalométriques sur des clichés radiographiques argentiques ou numériques, réalisés à $1 \mathrm{~m} 50$ ou à $4 \mathrm{~m}$. Les résultats montrent qu'il n'existe pas de différence statistiquement significative liée à la distance foyer-sujet ou au type de récepteur. Indépendamment du mode d'acquisition radiologique, chaque point céphalométrique présente un nuage de dispersion caractéristique, les points les plus fiables étant généralement situés dans le plan sagittal médian. Les mesures céphalométriques qui présentent la plus forte variabilité sont liées à la position des apex incisifs, ce qui conduit à relativiser leur importance lors de l'établissement du plan de traitement et du suivi thérapeutique. De manière analogue, l'utilisation du plan de Francfort est sujette à controverse au regard de la plus grande fiabilité des autres plans de référence du crâne et plus particulièrement S-Na.

ABSTRACT - Measurement errors in 2D cephalometrics. The objective of this study is to analyze the influence that two parameters, the source-subject distance and the type of receptor, have on the precision of localization of the 19 most frequently employed cephalometric landmark points and then to analyze the repercussions of localization errors on cephalometric measurements. The correct use of profile cephalograms (norma lateralis) for clinical purposes depends essentially on the precision of the identification and localization of the different landmark points that are required for the elaboration of the various cephalometric analyses. For this study we asked 53 orthodontists, selected at random, to identify 19 cephalometric points on standard profile or digitized films taken at distances of $1 \mathrm{~m} 50$ or of $4 \mathrm{~m}$. The results we obtained show no statistically significant differences in relation to the source-subject distance or to the type of receptor. Each cephalometric landmark presented a characteristic dispersion cloud; the most reliable points were primarily located on the median sagittal plane. The cephalometric measurements that demonstrated the greatest variability were associated with positions of the incisor apices, which makes their value doubtful in the establishment of treatment plans and in ongoing evaluations of treatment progress. For similar reasons, the worth of the Frankfort plane can be questioned when it is compared to other, much more reliable cranial reference planes especially S-Na.

\section{Introduction}

Les principes de la téléradiographie comme moyen de diagnostic en orthopédie dento-faciale ont été décrits dès 1922 par Carrera [7]. Mais c'est Broadbent, recherchant une méthode cliniquement utilisable permettant d'analyser les modifications dentoalvéolo-squelettiques liées à la croissance, aux théra-

\footnotetext{
* Auteur pour correspondance : foucart@univ-paris-diderot.fr
}

peutiques orthodontiques ou orthopédiques, qui jeta les bases de la céphalométrie "moderne » [8]. Le système de positionnement (céphalostat) qu'il met au point dès 1926, mais qu'il ne publie qu'en 1931 dans l'Angle Orthodontist, permet en effet une standardisation des clichés, essentielle aux applications cliniques et de recherche de la céphalométrie. La tête du patient, immobilisée dans une position standardisée, permet d'obtenir des clichés reproductibles et superposables présentant un agrandissement constant et faible (3\% 
à $5 \mathrm{~m}, 3,5 \%$ à $4 \mathrm{~m}$ et $11,5 \%$ à $1,50 \mathrm{~m}$ ), garanti par une grande distance foyer-sujet associée à une faible distance sujet-film [7,33].

Les possibilités de relevé de mesures angulaires et linéaires deviennent alors évidentes et confèrent à la céphalométrie un statut d'outil de diagnostic et de recherche orthodontique de premier plan.

Cependant, de nombreux auteurs ont rapidement observé que le relevé des mesures, secondaire à l'identification de repères céphalométriques, pouvait souffrir de trois grands types d'erreurs diminuant la fiabilité de cet examen. Savara et Tracey, puis Baumrind et Frantz, les ont définis comme les erreurs de projection, les erreurs de tracé et les erreurs de mesure $[4,5,26]$.

\subsection{Les erreurs de projection}

Les erreurs de projection sont liées à la réalisation du cliché. Baumrind et Frantz regroupent dans cette catégorie les erreurs de positionnement du sujet dans l'installation radiologique, celles liées à l'« écrasement » sur un film plan de structures anatomiques en trois dimensions, à la capacité du récepteur de l'image latente de reproduire fidèlement et avec une définition satisfaisante les différentes structures [4].

Les effets du positionnement dans le céphalostat ont été étudiés par Ahlqvist, Spolyar et Yoon [2, 28, 33]. Spolyar analyse les effets d'une rotation de la tête par rapport à la perpendiculaire au foyer radiologique. Les résultats qu'il présente indiquent que, dans $85 \%$ des cas, il existe une légère rotation de la tête (en moyenne de $1,87^{\circ}$ par rapport à l'axe perpendiculaire) et qu'une inclinaison de $5^{\circ}$ entraîne des modifications significatives dans les mesures linéaires de plus de $10 \mathrm{~mm}$. Ahlqvist constate qu'une rotation de $5^{\circ}$ de la tête du sujet entraîne une distorsion dans les mesures angulaires, qui peut être minimisée par l'utilisation d'une grande distance foyer-objet. Yoon, dans une étude portant sur la téléradiographie de face, observe que les repères antérieurs à l'axe de rotation se déplacent dans le sens de la rotation, ceux situés postérieurement en sens inverse. Ces résultats peuvent être extrapolés à la téléradiographie de profil : une inclinaison de la tête va faiblement affecter les structures situées sur l'axe sagittal médian, tandis que les structures latérales se déplaceront d'autant plus qu'elles seront éloignées de l'axe de rotation.

Hsiao étudie les déformations liées à la projection des structures en trois dimensions sur un film plan, en téléradiographie de face. L'étude porte sur le relevé de distances entre des points radio-opaques placés sur crâne sec puis sur les clichés obtenus après radiographie à $1,50 \mathrm{~m}$ : les résultats ne mettent pas en évidence de différence significative entre les mesures, qui présentent des variations inférieures à 0,5 millimètres après intégration d'un facteur d'agrandissement de 11,10 [17].

Björk et Mitgärd observent que la finesse et la qualité de l'image, mais également la complexité anatomique, influent sur la précision de lecture [6, 22]. Broch, analysant la précision de localisation, conclut que celle-ci dépend de critères tels que les caractéristiques anatomiques propres au sujet radiographié et leur superposition, la qualité du cliché, le flou de la structure à localiser lié au mouvement de la tête du sujet lors de la réalisation de l'examen (flou cinétique) [9].

Geelen ainsi que Lim étudient la précision d’identification sur film argentique et numérique. Ils n'observent pas de différence significative entre les deux types de récepteurs, mais la diminution de la dose d'irradiation permise par le capteur numérique leur fait conseiller l'utilisation préférentielle de ce dernier [13, 20]. Geelen complète son étude par une analyse du cliché numérique lu sur un écran d'ordinateur, en plus de la lecture de celui issu du reprographe : les résultats indiquent que la lecture sur écran est moins fiable que sur film. Seki et Okano font analyser des clichés numériques en faisant varier les constantes radiologiques : un cliché est réalisé avec les constantes classiques pour un film argentique, les autres avec une diminution d'exposition de 10, 20,50\%. L'étude statistique ne démontre pas de différence significative lors de l'identification entre les quatre types de clichés. Pour l'auteur, l'intérêt de l'emploi de capteurs numériques en termes de radioprotection apparaît évident [27].

Cohen, après avoir rappelé l'importance de la finesse et du contraste d'une radiographie, fait tracer, tout comme Macri et Wenzel, des clichés argentiques et numérisés secondairement sur ordinateur [10,21]. Macri effectue la numérisation à des résolutions différentes (haute/moyenne). Ces auteurs obtiennent des résultats identiques à ceux de Geelen : la lecture des clichés originaux est plus fiable que celle sur écran. Macri précise qu'il n'existe pas de différence statistiquement significative, fonction de la qualité de numérisation.

\subsection{Les erreurs de tracé}

Les erreurs de tracé, d'après Baumrind et Frantz, sont liées à l'identification par un observateur de 
repères anatomiques, dont certains sont situés dans des zones de faible contraste ou présentant une complexité anatomique particulière [4].

Mitgärd constate que l'expérience de l'observateur et sa connaissance précise de la définition du repère à identifier sont importantes [22]. Ces résultats sont nuancés par Wah qui n'observe pas de différence en fonction de l'expérience et l'entraînement des observateurs, mais plutôt en fonction de leur interprétation individuelle de la définition du point à localiser [32]. Toutefois, sa conception de l'entraînement et de l'expérience apparaît critiquable, puisque basée sur l'année de spécialité (de 1 à 3 ans) de l'observateur. Savage demande à douze orthodontistes, organisés en trois groupes par niveau d'expérience, de localiser 18 repères céphalométriques sur quatre clichés réalisés à 1,65 m [25]. Son étude ne révèle pas de différence dans la précision d'identification entre les trois groupes mais, de même que pour Wah, les critères d'évaluation de l'expérience restent très subjectifs. Les résultats obtenus par Savage lui permettent de proposer un classement des points en fonction de leur variabilité (dans l'ordre décroissant S, Po, Me, Or). L'auteur n'observe pas de différence statistiquement significatives entre les points construits et osseux.

Baumrind et Frantz, tout comme Broch, Richardson, Staburn et Vincent, ont analysé la fiabilité de l'identification de points régulièrement utilisés en céphalométrie [4, 9, 24, 29,31]. Ces différentes études présentent des similitudes dans les points étudiés et les résultats obtenus, malgré des différences dans le nombre de clichés analysés (de 10 à 100), d'observateurs (de 1 à 5) et de relevés (de 1 à 5 fois). Baumrind et Frantz observent que tous les points n'ont pas la même amplitude d'erreur, et que chaque point présente un nuage de dispersion caractéristique et généralement non circulaire. De plus, les nuages des points épousent le plus souvent les contours des structures anatomiques sur lesquelles ils sont situés.

Broch obtient des conclusions proches de celles de Baumrind et précise que la fiabilité dépend de cinq critères : de la qualité du cliché, de l'existence d'un flou cinétique, mais également de la précision de la méthode de relevé et de l'expérience de l'observateur. Le classement des points qu'il propose place les points médians et souffrant de peu de superpositions anatomiques aux premiers rangs (bords libres incisifs supérieurs et inférieurs, S, Na, Me, ENA, Pog, A).

Richardson observe que certains points sont plus fiables que d'autres, que certains se distribuent plutôt sur l'axe des X, d'autres sur l'axe des Y. Le classement des repères proposés dans cette étude identifie comme les plus fiables les points $\mathrm{S}, \mathrm{Me}$, ENA puis $\mathrm{Na}$, les moins fiables Bolton, Ophistion, Or et Go.

Staburn, dans une étude portant sur 14 repères et plus précisément l'apex de l'incisive inférieure, conclut comme les autres auteurs que la dispersion de chaque point n'est pas aléatoire, mais se fait au contraire de manière caractéristique. L'apex de l'incisive inférieure n'a pas, selon cet auteur, un haut niveau de reproductibilité, sa localisation étant incertaine dans $75 \%$ des cas. L'importance attribuée à la mesure de l'axe de l'incisive inférieure doit donc, selon Staburn, être fortement relativisée.

Vincent reprend les mêmes conclusions que les auteurs précédemment cités et constate que les points situés sur des courbures à faible progression sont les moins fiables, de même pour ceux situés dans des zones de faible contraste. L'auteur insiste également sur la nécessité de fournir une définition très précise du point, ne laissant pas de place pour une interprétation subjective.

Les résultats des cinq publications précédemment citées ont été compilés dans une méta-analyse par Trpkova, portant sur 15 repères céphalométriques [30]. Les conclusions de l'auteur sont qu'une erreur de 0,59 mm sur l'axe des $X$ et de 0,56 mm sur l'axe des $Y$ sont des niveaux acceptables de précision. Hägg, Cooke et Lau, dans une étude portant sur une analyse de la reproductibilité de repères céphalométriques non pas sur un sujet « vivant » mais sur crâne sec, obtiennent un classement des points les plus précis, proches de ceux retrouvés dans les différentes publications [14]. Les auteurs observent que l'absence de tissus mous diminue par quatre la valeur des déviations standard des erreurs de mesure; les points les plus affectés par la présence de tissus mous étant $\mathrm{Na}$ et ENA et pour les angles SNA et SNB.

Adenwalla publie une étude ciblée sur les points céphalométriques adjacents au condyle ; les résultats présentés indiquent que le porion et le condylion ne peuvent être localisés de manière précise sur des téléradiographies de profil en occlusion [1]. Selon l'auteur, leur identification fiable ne peut être réalisée que par superposition d'un cliché « bouche ouverte » avec un cliché « en occlusion ».

Cook et Gravely focalisent leur étude sur les structures mandibulaires utilisées dans la méthode de superposition décrite par Björk; les résultats les conduisent à conclure que la zone la plus fiable radiologiquement est la symphyse mandibulaire, que les erreurs sont moins importantes dans le sens horizontal 
que vertical et que les relevés des structures médianes sont plus précis que ceux des structures bilatérales [11].

Haynes et Chau effectuent une analyse inter- et intra-observateur portant sur des repères céphalométriques conventionnels (S, ENA, Na, A, B, Me, Go et $\mathrm{Ba}$ ) et d'autres spécifiques à l'analyse de Delaire. Les résultats mettent en évidence une reproductibilité bien supérieure en intra-observateur qu'en interobservateur, ainsi qu'une plus grande difficulté à localiser les points situés sur des corticales osseuses à concavité ou convexité faible [15].

\subsection{Les erreurs de mesure}

Les erreurs de mesure sont liées, selon Baumrind, à l'erreur ou à l'imprécision de relevé de mesures angulaires et linéaires par l'observateur [4,5].

Dans la deuxième partie de son étude sur la précision de lecture des téléradiographies, l'auteur observe que les erreurs de mesures sont dépendantes des erreurs de localisation des points. Ainsi, les lignes reliant les points les moins fiables aux nuages de dispersion les plus étendus présentent logiquement les variations les plus importantes. De plus, la distance séparant les deux points déterminant un plan ou un segment de ligne a une influence: la précision sera d'autant plus grande que la distance sera importante. Enfin, toujours selon Baumrind, la fiabilité des mesures dépend de la façon dont les segments de ligne reliant les différents points rencontrent les nuages de ces derniers. L'auteur propose, afin d'augmenter la précision des mesures linéaires et angulaires, de répéter les relevés.

Richardson, Foster ainsi que Pancherz [12, 23, 24] ont analysé les variations lors de la mesure de lignes de référence. Ces auteurs obtiennent des conclusions assez proches : la ligne de référence la plus fiable et présentant le moins de variations est la ligne S-Na. Richardson analyse les plans S-Na, Francfort, maxillaire, mandibulaire et conclut que les lignes et plans les plus fiables sont ceux reliant les points céphalométriques présentant le moins de variations. Le classement proposé par l'auteur est dans l'ordre : S-Na, mandibulaire, maxillaire, de De Coster et Francfort avant dernier, devant le plan optique.

Foster étudie les rapports de ces mêmes plans entre eux et par rapport à l'horizontal ; il conclut que, bien que le plan de Francfort et le plan maxillaire soient presque parallèles entre eux et par rapport à l'horizontal, les écarts de variations pour tous les autres plans restent considérables. Le plan S-Na apparaît comme le plus précisément reproduit. Toujours selon Foster, les variations des rapports des différents plans entre eux sont plus importantes que celles causées par les erreurs de positionnement de la tête du sujet.

Les objectifs de notre étude sont :

- d'analyser l'influence du récepteur, argentique ou numérique, sur l'erreur de mesure ;

- d'analyser l'influence de la distance foyer-sujet, $4 \mathrm{~m}$ versus 1,5 m, sur l'erreur de mesure ;

- de déterminer quels sont les points céphalométriques qui peuvent être considérés comme les plus fiables, d'après l'analyse statistique de leurs nuages de dispersion ;

- de déterminer quelles sont les mesures linéaires et angulaires qui peuvent être considérées comme les plus reproductibles.

\section{Matériel et méthode}

\subsection{Matériel}

Quatre clichés téléradiographiques en norma latéralis ont été réalisés sur un même sujet en occlusion d'intercuspidation maximale et ne présentant pas de dysmorphose (classe I molaire et canine, sans encombrement dentaire).

Les clichés ont été réalisés sur deux « installations » radiologiques, l'une à $4 \mathrm{~m}$ (CGR Pulsatrix, $388 \mathrm{~cm}, 19 \mathrm{~cm}$ ), l'autre à 1,50 m (Orthophos XG 5, $150 \mathrm{~cm}, 16 \mathrm{~cm}$ ), en utilisant deux récepteurs différents, l'un argentique (Agfa Curis Blue HC-S plus), l'autre numérique (Philips PCR AC 5000).

Avant la réalisation des clichés, chaque installation a bénéficié d'un contrôle qualité tant au niveau du générateur que du céphalostat (erreur d'alignement des olives auriculaires par rapport au rayon directeur du faisceau principal inférieur au millimètre). Le porte-cassette a été équipé d'une plaque de plastique comportant quatre repères radio-opaques, placés devant la cassette contenant le récepteur de l'image latente.

Le sujet, bénéficiant des mesures de radioprotection appropriées, a été positionné dans le céphalostat par un manipulateur expérimenté en radiodiagnostic. Le plan sagittal médian de la tête du sujet a été placé perpendiculairement au rayon principal du tube et le plan de Francfort orienté parallèlement au plan horizontal de l'installation. Les clichés argentiques et numériques ont été successivement réalisés, le sujet restant immobile lors du changement de capteur. Les constantes utilisées ont été optimisées afin de respecter le principe de radioprotection «ALARA » 
( As Low As Reasonably Achievable ») énoncé dans les directives EURATOM (96/29 et 97/43).

Chaque combinaison « distance-type de récepteur » a permis de réaliser un cliché à :

- 4 m associé à une chaîne de développement argentique,

- 4 m associé à une chaîne de développement numérique,

- à 1,50 m associé à une chaîne de développement argentique,

- à 1,50 m associé à une chaîne de développement numérique.

\subsection{Méthode}

\subsubsection{Recueil des données}

Un groupe de 70 orthodontistes exclusifs a été aléatoirement sélectionné durant les Journées de l'Orthodontie. Un questionnaire leur a été confié, ainsi qu'une introduction aux buts de l'étude. Les participants ont été assurés de l'anonymat de leurs réponses. Un seul rappel a été effectué et 53 orthodontistes (75,71\%) ont accepté de répondre au questionnaire et de localiser 19 points céphalométriques sur les quatre types de clichés.

Ces quatre téléradiographies, au format standard $24 \times 30 \mathrm{~cm}$, ne présentaient aucune identification à l'exception des repères radio-opaques. L'identification des points a été facilitée par l'utilisation d'un négatoscope, identique pour tous les observateurs (Slim Lite Lightbox : luminosité $1,800 \mathrm{~cd} / \mathrm{mm}^{2}$, température de couleur $5.300 \mathrm{~K}$ ). Chaque participant a effectué le test assis, dans un environnement calme, le négatoscope placé face à lui sur une table. Les quatre types de clichés ont été présentés dans un ordre aléatoire.

Les points céphalométriques étudiés ont été sélectionnés parmi ceux les plus fréquemment requis dans les analyses céphalométriques les plus usitées. Les définitions précises des points ont été fournies à chaque participant, dans un ordre horaire partant du point $\mathrm{S}$ et se finissant au point Ophistion.

Les points à localiser étaient :

- des points squelettiques médians: S, Na, ENA, ENP, A, B, Pm, Pog, Me, Ba, Op ;
- des points squelettiques bilatéraux : Or, Go, Ar, Po ;

- des points dentaires: apex incisive maxillaire, bord libre incisive maxillaire, apex incisive mandibulaire, bord libre incisive mandibulaire.

Les participants avaient la possibilité de ne pas tracer les points qu'ils ne connaissaient pas ou qu'ils n'utilisaient pas régulièrement. Aucune limite de temps ou de méthode d'analyse n'a été imposée pour réaliser l'ensemble du test.

\subsubsection{Analyse des données}

Les tracés ont été numérisés à l'aide d'un scanner à plat Epson Perfection 2450 Photo sur lequel avait été transférée la position des quatre repères radioopaques solidaires de l'installation radiologique. Chaque tracé a été repositionné selon ces repères, puis numérisé à une définition de 150 pixels/pouce, assurant une précision au dixième de millimètre. Les tracés numérisés ont ensuite été intégrés dans le logiciel Adobe Photoshop par l'observateur principal. Les images obtenues ont été traitées par un logiciel d'analyse d'image développé sur Virtual Basic.

Afin d'éliminer l'erreur liée à la numérisation des clichés, sur chaque tracé, les coordonnées des quatre repères radio-opaques ont été initialement relevées par le logiciel d'analyse d'image afin de servir de référence au système orthonormé d'identification des coordonnées.

Les coordonnées $X$ et $Y$ des 19 points relevés ont été compilées dans une feuille de calcul générée par le logiciel Excel. La moyenne arithmétique, l'écart type ainsi que la dispersion de chacun des 19 points, sur les quatre types de clichés, ont été calculés. Les déviations sur l'axe des $X$ et des $Y$ des 19 points ont d'abord été analysées séparément, puis combinées dans une ellipse afin d'analyser la surface de dispersion de chaque point au moyen d'une valeur unique.

A partir de ces points relevés, les mesures céphalométriques non construites (20 mesures angulaires et 11 mesures linéaires) des six analyses les plus couramment utilisées ont été effectuées (analyses de Tweed, Steiner, Ricketts, MacNamara, Downs, Ballard). Leurs valeurs ont été traduites à l'aide d'équations dont l'une est reportée en exemple ci-dessous :

$$
F M I A=\cos ^{-1}\left(\frac{\left(x_{B i}-x_{A i}\right)\left(x_{O r}-x_{P}\right)+\left(y_{B i}-y_{A i}\right)\left(y_{O r}-y_{P}\right)}{\sqrt{\left(x_{B i}-x_{A i}\right)^{2}+\left(y_{B i}-y_{A i}\right)^{2}} \times \sqrt{\left(x_{O r}-x_{P}\right)^{2}+\left(y_{O r}-y_{P}\right)^{2}}}\right)
$$




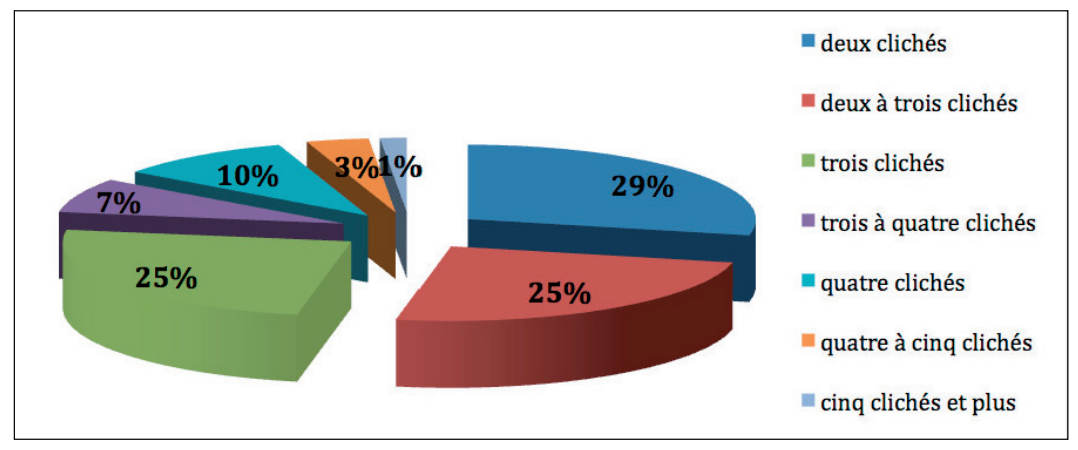

Figure 1

Nombre moyen de clichés téléradiographiques réalisés durant un traitement d'orthodontie.
L'approche statistique a été réalisée à l'aide du test $T$-student pour variables appairées dans le logiciel Statview. L'hypothèse nulle a été rejetée si $p>0,05$.

\section{Résultats}

Sur l'ensemble des 70 praticiens exclusifs sollicités pour participer à l'étude, 53 ont accepté $(75,7 \%)$ et 17 ont refusé $(24,3 \%)$. L'analyse des résultats des questionnaires révèle :

- un sexe ratio de 28 femmes (52,8\%) pour 25 hommes $(47,2 \%)$;

- une durée moyenne de pratique orthodontique de 17,5 ans ;

- que $100 \%$ des praticiens déclarent utiliser des clichés téléradiographiques de profil lors de leur pratique orthodontique ;

- que $83 \%$ déclarent effectuer systématiquement une analyse céphalométrique, 11,5\% souvent et 5,5\% parfois ;

- que les résultats fournis par l'analyse céphalométrique ont une place jugée « très importante » dans le diagnostic clinique pour $20,5 \%$ des praticiens, « importante » pour $70 \%$, «peu importante» pour $8 \%$ et « accessoire » pour un seul d'entre eux ;

- que les types d'analyses pratiquées sont : l'analyse de Tweed pour 40 praticiens $(75,5 \%)$, l'analyse de Ricketts pour 19 d'entre eux (35,8 \%), l'analyse de Steiner pour 10 (18,9\%), l'analyse de Delaire pour 4 (7,6\%), les analyses de Sassouni et de McNamara pour 3 (5,7 \%) et autres pour 4 d'entre eux (les praticiens ayant la possibilité de cocher plusieurs propositions, le total des réponses est supérieur à $100 \%$ ) ;

- que le nombre de téléradiographies utilisées, en moyenne, au cours d'un traitement d'orthodontie est de deux pour $29 \%$ des praticiens interrogés, entre deux et trois pour $25 \%$, de trois pour $25 \%$, de trois à quatre pour $7 \%$, de quatre pour $10 \%$, de plus de quatre pour $4 \%$ (Fig. 1).
Les tableaux 1 et 2 présentent les écarts-types, les intervalles de confiance ainsi que les surfaces de dispersion, relevés en $X$ et $Y$ pour chaque distance utilisée en fonction du type de récepteur. L'étude statistique montre qu'il n'existe pas de différence statistiquement significative d'identification liée au récepteur $(p>$ $0,99)$.

Le tableau 3 présente l'écart-type et l'intervalle de confiance de chaque point en fonction de la distance utilisée. L'étude statistique montre qu'il n'existe pas de différence statistiquement significative d'identification liée à la distance $(p>0,99)$.

Le tableau 4 compile l'écart-type de chaque point sur les axes $X$ et $Y$, mais également leur surface de dispersion, en millimètres carrés. Ces surfaces sont également présentées sous formes d'ellipses dont les rayons sont caractérisés par les valeurs des déviations standard selon $X$ et $Y$. Chaque point est classé en fonction de la surface de son nuage de dispersion. La figure 2 projette sur le cliché de référence la dispersion des points réellement identifiés.

Le tableau 5 présente la moyenne, l'écart-type et le classement moyen aux 4 types de clichés, pour les 20 mesures angulaires et les 11 mesures linéaires calculées.

\section{Discussion}

En 2011, l'Ordre National des ChirurgiensDentistes recensait près de 2000 spécialistes. Dans la population des chirurgiens-dentistes, l'âge moyen était de 48 ans et le sexe ration en faveur des hommes de 6:4. Par rapport aux données de l'Ordre, le sexe ratio (10:9) et la durée moyenne d'exercice, rapportée à l'âge, semblent souligner la représentativité de l'échantillon.

Les types d'analyses pratiqués par les participants confirment le choix des pointsétudiés. Le questionnaire révèle l'attachement des praticiens aux analyses céphalométriques. 
Tableau 1

Écart-type, intervalle de confiance et surface de dispersion de chaque point, pour les clichés réalisés à $4 \mathrm{~m}$.

\begin{tabular}{|c|c|c|c|c|c|c|c|}
\hline \multirow{2}{*}{$\begin{array}{l}\text { Coordonnées } \\
\text { Points }\end{array}$} & \multicolumn{3}{|c|}{$4 \mathrm{~m}$ - argentique } & \multicolumn{3}{|c|}{$4 \mathrm{~m}$ - numérique } & \multirow[t]{2}{*}{$p$} \\
\hline & ET & IC & $\mathrm{S}\left(\mathrm{mm}^{2}\right)$ & ET & IC & $\mathrm{S}\left(\mathrm{mm}^{2}\right)$ & \\
\hline$x S$ & 0,04 & 0,18 & \multirow{2}{*}{0,754} & 0,04 & 0,21 & \multirow{2}{*}{0,628} & $>0,99 \mathrm{NS}$ \\
\hline$y S$ & 0,06 & 0,28 & & 0,05 & 0,22 & & $>0,99 \mathrm{NS}$ \\
\hline$x \mathrm{Na}$ & 0,04 & 0,25 & \multirow{2}{*}{0,88} & 0,04 & 0,22 & \multirow{2}{*}{0,88} & $>0,99 \mathrm{NS}$ \\
\hline$y \mathrm{Na}$ & 0,07 & 0,34 & & 0,07 & 0,28 & & $>0,99 \mathrm{NS}$ \\
\hline$x \mathrm{Or}$ & 0,17 & 0,82 & \multirow{2}{*}{4,273} & 0,2 & 0,96 & \multirow{2}{*}{7,54} & $>0,99 \mathrm{NS}$ \\
\hline$y \mathrm{Or}$ & 0,08 & 0,43 & & 0,12 & 0,67 & & $>0,99 \mathrm{NS}$ \\
\hline$x$ ENA & 0,05 & 0,21 & \multirow{2}{*}{0,785} & 0,07 & 0,38 & \multirow{2}{*}{1,539} & $>0,99 \mathrm{NS}$ \\
\hline y ENA & 0,05 & 0,29 & & 0,07 & 0,35 & & $>0,99 \mathrm{NS}$ \\
\hline$x \mathrm{ENP}$ & 0,41 & 1,75 & \multirow{2}{*}{9,016} & 0,47 & 1,75 & \multirow{2}{*}{11,81} & $>0,99 \mathrm{NS}$ \\
\hline$y$ ENP & 0,07 & 0,33 & & 0,08 & 0,43 & & $>0,99 \mathrm{NS}$ \\
\hline$x \mathrm{~A}$ & 0,03 & 0,12 & \multirow{2}{*}{0,942} & 0,03 & 0,2 & \multirow{2}{*}{1,131} & $>0,99 \mathrm{NS}$ \\
\hline y A & 0,1 & 0,51 & & 0,12 & 0,44 & & $>0,99 \mathrm{NS}$ \\
\hline$x$ IS apex & 0,13 & 0,67 & \multirow{2}{*}{4,901} & 0,13 & 0,71 & \multirow{2}{*}{5,718} & $>0,99 \mathrm{NS}$ \\
\hline$y$ IS apex & 0,12 & 0,69 & & 0,14 & 0,64 & & $>0,99 \mathrm{NS}$ \\
\hline$x$ IS bl & 0,03 & 0,13 & \multirow{2}{*}{0,471} & 0,03 & 0,17 & \multirow{2}{*}{0,565} & $>0,99 \mathrm{NS}$ \\
\hline$y$ IS bl & 0,05 & 0,27 & & 0,06 & 0,43 & & $>0,99 \mathrm{NS}$ \\
\hline$x$ II bl & 0,05 & 0,2 & \multirow{2}{*}{0,942} & 0,06 & 0,24 & \multirow{2}{*}{0,942} & $>0,99 \mathrm{NS}$ \\
\hline y II bl & 0,06 & 0,28 & & 0,05 & 0,24 & & $>0,99 \mathrm{NS}$ \\
\hline$x$ II apex & 0,16 & 0,59 & \multirow{2}{*}{9,55} & 0,15 & 0,49 & \multirow{2}{*}{8,011} & $>0,99 \mathrm{NS}$ \\
\hline y II apex & 0,19 & 0,76 & & 0,17 & 0,68 & & $>0,99 \mathrm{NS}$ \\
\hline$x \mathrm{~B}$ & 0,03 & 0,1 & \multirow{2}{*}{1,791} & 0,03 & 0,17 & 1508 & $>0,99 \mathrm{NS}$ \\
\hline$y B$ & 0,19 & 1,14 & & 0,16 & 0,81 & 1,508 & $>0,99 \mathrm{NS}$ \\
\hline$x \mathrm{Pm}$ & 0,04 & 0,16 & & 0,01 & 0,3 & 0601 & $>0,99 \mathrm{NS}$ \\
\hline y $\mathrm{Pm}$ & 0,22 & 1,27 & 2,105 & 0,22 & 1,18 & 0,091 & $>0,99 \mathrm{NS}$ \\
\hline$x \operatorname{Pog}$ & 0,03 & 0,17 & & 0,04 & 0,18 & & $>0,99 \mathrm{NS}$ \\
\hline$y \operatorname{Pog}$ & 0,18 & 0,88 & 1,090 & 0,17 & 0,7 & 2,136 & $>0,99 \mathrm{NS}$ \\
\hline$x \mathrm{Me}$ & 0,08 & 0,4 & $\cap 005$ & 0,08 & 0,36 & 1257 & $>0,99 \mathrm{NS}$ \\
\hline$y \mathrm{Me}$ & 0,04 & 0,22 & 0,005 & 0,05 & 0,24 & $1,2 J$ & $>0,99 \mathrm{NS}$ \\
\hline$x \mathrm{Go}$ & 0,3 & 1,02 & 377 & 0,29 & 1,11 & 3553 & $>0,99 \mathrm{NS}$ \\
\hline$y \mathrm{Go}$ & 0,4 & 1,24 & 31,1 & 0,39 & 1,28 & 35,35 & $>0,99 \mathrm{NS}$ \\
\hline$x \mathrm{Ar}$ & 0,09 & 0,65 & 2362 & 0,09 & 0,69 & 2827 & $>0,99 \mathrm{NS}$ \\
\hline$y \mathrm{Ar}$ & 0,08 & 0,48 & $2, \angle O 2$ & 0,1 & 0,61 & $2,8<1$ & $>0,99 \mathrm{NS}$ \\
\hline$x \mathrm{Ba}$ & 0,35 & 1,86 & & 0,29 & 1,93 & & $>0,99 \mathrm{NS}$ \\
\hline$y \mathrm{Ba}$ & 0,26 & 1,28 & 28,59 & 0,22 & 1,02 & 20,04 & $>0,99 \mathrm{NS}$ \\
\hline$x$ Por & 0,21 & 1,73 & & 0,12 & 0,7 & 1 & $>0,99 \mathrm{NS}$ \\
\hline$y$ Por & 0,1 & 0,68 & 0,591 & 0,13 & 1,09 & 4,901 & $>0,99 \mathrm{NS}$ \\
\hline$x \mathrm{Op}$ & 0,93 & 3,5 & 5250 & 1,02 & 3,44 & 64088 & $>0,99 \mathrm{NS}$ \\
\hline y Op & 0,18 & 0,86 & 32,59 & 0,2 & 0,93 & 04,080 & $>0,99 \mathrm{NS}$ \\
\hline $\begin{array}{l}X \text { MOY } \\
Y \text { MOY }\end{array}$ & $\begin{array}{l}0,108 \\
0,087\end{array}$ & $\begin{array}{l}- \\
-\end{array}$ & 2,958 & $\begin{array}{l}0,104 \\
0,086\end{array}$ & $\begin{array}{l}- \\
-\end{array}$ & 2,812 & $\begin{array}{l}- \\
-\end{array}$ \\
\hline
\end{tabular}


Tableau 2

Écart-type, intervalle de confiance et surface de dispersion de chaque point, pour les clichés réalisés à 1,5 m.

\begin{tabular}{|c|c|c|c|c|c|c|c|}
\hline \multirow{2}{*}{$\begin{array}{l}\text { Coordonnées } \\
\text { Points }\end{array}$} & \multicolumn{3}{|c|}{$1,5 \mathrm{~m}-$ argentique } & \multicolumn{3}{|c|}{$1,5 \mathrm{~m}$ - numérique } & \multirow[t]{2}{*}{$p$} \\
\hline & ET & IC & $\mathrm{S}\left(\mathrm{mm}^{2}\right)$ & ET & IC & $\mathrm{S}\left(\mathrm{mm}^{2}\right)$ & \\
\hline$x \mathrm{~S}$ & 0,05 & 0,23 & \multirow{2}{*}{0,785} & 0,04 & 0,22 & \multirow{2}{*}{0,628} & $>0,99 \mathrm{NS}$ \\
\hline$y S$ & 0,05 & 0,21 & & 0,05 & 0,22 & & $>0,99 \mathrm{NS}$ \\
\hline$x \mathrm{Na}$ & 0,05 & 0,23 & \multirow{2}{*}{1,1} & 0,05 & 0,27 & \multirow{2}{*}{1,1} & $>0,99 \mathrm{NS}$ \\
\hline$y \mathrm{Na}$ & 0,07 & 0,32 & & 0,07 & 0,51 & & $>0,99 \mathrm{NS}$ \\
\hline$x$ Or & 0,24 & 0,94 & \multirow{2}{*}{15,08} & 0,29 & 1,27 & \multirow{2}{*}{16,4} & $>0,99 \mathrm{NS}$ \\
\hline$y \mathrm{Or}$ & 0,2 & 0,68 & & 0,18 & 0,87 & & $>0,99 \mathrm{NS}$ \\
\hline$x$ ENA & 0,04 & 0,15 & \multirow{2}{*}{0,377} & 0,05 & 0,22 & \multirow{2}{*}{0,628} & $>0,99 \mathrm{NS}$ \\
\hline$y$ ENA & 0,03 & 0,13 & & 0,04 & 0,17 & & $>0,99 \mathrm{NS}$ \\
\hline$x$ ENP & 0,45 & 1,66 & \multirow{2}{*}{5,655} & 0,42 & 1,47 & \multirow{2}{*}{6,597} & $>0,99 \mathrm{NS}$ \\
\hline$y$ ENP & 0,04 & 0,21 & & 0,05 & 0,23 & & $>0,99 \mathrm{NS}$ \\
\hline$x \mathrm{~A}$ & 0,03 & 0,18 & \multirow{2}{*}{0,754} & 0,03 & 0,12 & \multirow{2}{*}{0,754} & $>0,99 \mathrm{NS}$ \\
\hline$y \mathrm{~A}$ & 0,08 & 0,43 & & 0,08 & 0,51 & & $>0,99 \mathrm{NS}$ \\
\hline$x$ IS apex & 0,13 & 0,59 & \multirow{2}{*}{5,309} & 0,12 & 0,6 & \multirow{2}{*}{4,901} & $>0,99 \mathrm{NS}$ \\
\hline$y$ IS apex & 0,13 & 0,79 & & 0,13 & 0,71 & & $>0,99 \mathrm{NS}$ \\
\hline$x$ IS bl & 0,04 & 0,21 & \multirow{2}{*}{0,503} & 0,04 & 0,23 & \multirow{2}{*}{0,503} & $>0,99 \mathrm{NS}$ \\
\hline$y$ IS bl & 0,04 & 0,2 & & 0,04 & 0,2 & & $>0,99 \mathrm{NS}$ \\
\hline$x$ II bl & 0,06 & 0,23 & \multirow{2}{*}{0,942} & 0,07 & 0,24 & \multirow{2}{*}{1,1} & $>0,99 \mathrm{NS}$ \\
\hline$y$ II bl & 0,05 & 0,17 & & 0,05 & 0,24 & & $>0,99 \mathrm{NS}$ \\
\hline$x$ II apex & 0,11 & 0,55 & \multirow{2}{*}{6,912} & 0,1 & 0,46 & \multirow{2}{*}{5,655} & $>0,99 \mathrm{NS}$ \\
\hline$y$ II apex & 0,2 & 0,97 & & 0,18 & 0,84 & & $>0,99 \mathrm{NS}$ \\
\hline$x \mathrm{~B}$ & 0,04 & 0,29 & \multirow{2}{*}{1,382} & 0,03 & 0,15 & 1225 & $>0,99 \mathrm{NS}$ \\
\hline$y B$ & 0,11 & 0,64 & & 0,13 & 0,56 & $1,2 \angle J$ & $>0,99 \mathrm{NS}$ \\
\hline$x \mathrm{Pm}$ & 0,06 & 0,24 & 277 & 0,04 & 0,17 & 2011 & $>0,99 \mathrm{NS}$ \\
\hline$y \mathrm{Pm}$ & 0,2 & 1 & (1T & 0,16 & 0,68 & 2,011 & $>0,99 \mathrm{NS}$ \\
\hline$x$ Pog & 0,06 & 0,44 & 2302 & 0,04 & 0,2 & 202 & $>0,99 \mathrm{NS}$ \\
\hline$y \operatorname{Pog}$ & 0,18 & 0,94 & נצכ, & 0,18 & 0,74 & 2,202 & $>0,99 \mathrm{NS}$ \\
\hline$x \mathrm{Me}$ & 0,07 & 0,39 & 066 & 0,07 & 0,32 & 066 & $>0,99 \mathrm{NS}$ \\
\hline$y \mathrm{Me}$ & 0,03 & 0,13 & 0,00 & 0,03 & 0,14 & 0,00 & $>0,99 \mathrm{NS}$ \\
\hline$x \mathrm{Go}$ & 0,31 & 1,37 & 2480 & 0,28 & 1,31 & ד & $>0,99 \mathrm{NS}$ \\
\hline$y \mathrm{Go}$ & 0,35 & 1,14 & ( & 0,34 & 1,26 & 29,91 & $>0,99 \mathrm{NS}$ \\
\hline$x \mathrm{Ar}$ & 0,12 & 0,83 & 4520 & 0,09 & 0,53 & 545 & $>0,99 \mathrm{NS}$ \\
\hline$y \mathrm{Ar}$ & 0,12 & 0,66 & & 0,09 & 0,58 & & $>0,99 \mathrm{NS}$ \\
\hline$x \mathrm{Ba}$ & 0,25 & 1,37 & 1 & 0,2 & 0,88 & 1202 & $>0,99 \mathrm{NS}$ \\
\hline$y \mathrm{Ba}$ & 0,27 & 1,5 & 21,21 & 0,22 & 0,9 & 15,02 & $>0,99 \mathrm{NS}$ \\
\hline$x$ Por & 0,3 & 1,84 & 1) 21 & 0,21 & 1,29 & 2326 & $>0,99 \mathrm{NS}$ \\
\hline$y$ Por & 0,12 & 0,6 & ג1 & 0,14 & 0,69 & , & $>0,99 \mathrm{NS}$ \\
\hline$x \mathrm{Op}$ & 0,97 & 3,66 & 67041 & 0,92 & 3,71 & 66476 & $>0,99 \mathrm{NS}$ \\
\hline$y \mathrm{Op}$ & 0,22 & 1,18 & , & 0,23 & 0,84 & טוד, & $>0,99 \mathrm{NS}$ \\
\hline $\begin{array}{l}X \text { MOY } \\
\text { Y MOY }\end{array}$ & $\begin{array}{l}0,115 \\
0,084\end{array}$ & $\begin{array}{l}- \\
-\end{array}$ & 3,035 & $\begin{array}{c}0,099 \\
0,08\end{array}$ & $\begin{array}{l}- \\
-\end{array}$ & 2,487 & $\begin{array}{l}- \\
-\end{array}$ \\
\hline
\end{tabular}


Tableau 3

Écart-type et intervalle de confiance de chaque point en fonction de la distance.

\begin{tabular}{|c|c|c|c|c|c|c|c|c|c|c|}
\hline \multirow{2}{*}{$\begin{array}{l}\text { Coordonnées } \\
\text { Points }\end{array}$} & \multicolumn{2}{|c|}{$4 \mathrm{~m}$ argentique } & \multicolumn{2}{|c|}{$1,5 \mathrm{~m}$ argentique } & \multirow[t]{2}{*}{$p$} & \multicolumn{2}{|c|}{$4 \mathrm{~m}$ numérique } & \multicolumn{2}{|c|}{ 1,5 m numérique } & \multirow[t]{2}{*}{$p$} \\
\hline & ET & IC & ET & IC & & ET & IC & ET & IC & \\
\hline$x S$ & 0,04 & 0,18 & 0,05 & 0,23 & $>0,99 \mathrm{NS}$ & 0,04 & 0,21 & 0,04 & 0,22 & $>0,99 \mathrm{NS}$ \\
\hline$y S$ & 0,06 & 0,28 & 0,05 & 0,21 & $>0,99 \mathrm{NS}$ & 0,05 & 0,22 & 0,05 & 0,22 & $>0,99 \mathrm{NS}$ \\
\hline$x \mathrm{Na}$ & 0,04 & 0,25 & 0,05 & 0,23 & $>0,99 \mathrm{NS}$ & 0,04 & 0,22 & 0,05 & 0,27 & $>0,99 \mathrm{NS}$ \\
\hline$y \mathrm{Na}$ & 0,07 & 0,34 & 0,07 & 0,32 & $>0,99 \mathrm{NS}$ & 0,07 & 0,28 & 0,07 & 0,51 & $>0,99 \mathrm{NS}$ \\
\hline$x$ Or & 0,17 & 0,82 & 0,24 & 0,94 & $>0,99 \mathrm{NS}$ & 0,2 & 0,96 & 0,29 & 1,27 & $>0,99 \mathrm{NS}$ \\
\hline$y$ Or & 0,08 & 0,43 & 0,2 & 0,68 & $>0,99 \mathrm{NS}$ & 0,12 & 0,67 & 0,18 & 0,87 & $>0,99 \mathrm{NS}$ \\
\hline$x$ ENA & 0,05 & 0,21 & 0,04 & 0,15 & $>0,99 \mathrm{NS}$ & 0,07 & 0,38 & 0,05 & 0,22 & $>0,99 \mathrm{NS}$ \\
\hline$y$ ENA & 0,05 & 0,29 & 0,03 & 0,13 & $>0,99 \mathrm{NS}$ & 0,07 & 0,35 & 0,04 & 0,17 & $>0,99 \mathrm{NS}$ \\
\hline$x$ ENP & 0,41 & 1,75 & 0,45 & 1,66 & $>0,99 \mathrm{NS}$ & 0,47 & 1,75 & 0,42 & 1,47 & $>0,99 \mathrm{NS}$ \\
\hline$y$ ENP & 0,07 & 0,33 & 0,04 & 0,21 & $>0,99 \mathrm{NS}$ & 0,08 & 0,43 & 0,05 & 0,23 & $>0,99 \mathrm{NS}$ \\
\hline$x \mathrm{~A}$ & 0,03 & 0,12 & 0,03 & 0,18 & $>0,99 \mathrm{NS}$ & 0,03 & 0,2 & 0,03 & 0,12 & $>0,99 \mathrm{NS}$ \\
\hline$y \mathrm{~A}$ & 0,1 & 0,51 & 0,08 & 0,43 & $>0,99 \mathrm{NS}$ & 0,12 & 0,44 & 0,08 & 0,51 & $>0,99 \mathrm{NS}$ \\
\hline$x$ IS apex & 0,13 & 0,67 & 0,13 & 0,59 & $>0,99 \mathrm{NS}$ & 0,13 & 0,71 & 0,12 & 0,6 & $>0,99 \mathrm{NS}$ \\
\hline$y$ IS apex & 0,12 & 0,69 & 0,13 & 0,79 & $>0,99 \mathrm{NS}$ & 0,14 & 0,64 & 0,13 & 0,71 & $>0,99 \mathrm{NS}$ \\
\hline$x$ IS bl & 0,03 & 0,13 & 0,04 & 0,21 & $>0,99 \mathrm{NS}$ & 0,03 & 0,17 & 0,04 & 0,23 & $>0,99 \mathrm{NS}$ \\
\hline$y$ IS bl & 0,05 & 0,27 & 0,04 & 0,2 & $>0,99 \mathrm{NS}$ & 0,06 & 0,43 & 0,04 & 0,2 & $>0,99 \mathrm{NS}$ \\
\hline$x \mathrm{II} \mathrm{bl}$ & 0,05 & 0,2 & 0,06 & 0,23 & $>0,99 \mathrm{NS}$ & 0,06 & 0,24 & 0,07 & 0,24 & $>0,99 \mathrm{NS}$ \\
\hline y II bl & 0,06 & 0,28 & 0,05 & 0,17 & $>0,99 \mathrm{NS}$ & 0,05 & 0,24 & 0,05 & 0,24 & $>0,99 \mathrm{NS}$ \\
\hline$x$ II apex & 0,16 & 0,59 & 0,11 & 0,55 & $>0,99 \mathrm{NS}$ & 0,15 & 0,49 & 0,1 & 0,46 & $>0,99 \mathrm{NS}$ \\
\hline$y$ II apex & 0,19 & 0,76 & 0,2 & 0,97 & $>0,99 \mathrm{NS}$ & 0,17 & 0,68 & 0,18 & 0,84 & $>0,99 \mathrm{NS}$ \\
\hline$x \mathrm{~B}$ & 0,03 & 0,1 & 0,04 & 0,29 & $>0,99 \mathrm{NS}$ & 0,03 & 0,17 & 0,03 & 0,15 & $>0,99 \mathrm{NS}$ \\
\hline$y B$ & 0,19 & 1,14 & 0,11 & 0,64 & $>0,99 \mathrm{NS}$ & 0,16 & 0,81 & 0,13 & 0,56 & $>0,99 \mathrm{NS}$ \\
\hline$x \mathrm{Pm}$ & 0,04 & 0,16 & 0,06 & 0,24 & $>0,99 \mathrm{NS}$ & 0,01 & 0,3 & 0,04 & 0,17 & $>0,99 \mathrm{NS}$ \\
\hline$y \mathrm{Pm}$ & 0,22 & 1,27 & 0,2 & 1 & $>0,99 \mathrm{NS}$ & 0,22 & 1,18 & 0,16 & 0,68 & $>0,99 \mathrm{NS}$ \\
\hline$x$ Pog & 0,03 & 0,17 & 0,06 & 0,44 & $>0,99 \mathrm{NS}$ & 0,04 & 0,18 & 0,04 & 0,2 & $>0,99 \mathrm{NS}$ \\
\hline$y$ Pog & 0,18 & 0,88 & 0,18 & 0,94 & $>0,99 \mathrm{NS}$ & 0,17 & 0,7 & 0,18 & 0,74 & $>0,99 \mathrm{NS}$ \\
\hline$x \mathrm{Me}$ & 0,08 & 0,4 & 0,07 & 0,39 & $>0,99 \mathrm{NS}$ & 0,08 & 0,36 & 0,07 & 0,32 & $>0,99 \mathrm{NS}$ \\
\hline$y \mathrm{Me}$ & 0,04 & 0,22 & 0,03 & 0,13 & $>0,99 \mathrm{NS}$ & 0,05 & 0,24 & 0,03 & 0,14 & $>0,99 \mathrm{NS}$ \\
\hline$x$ Go & 0,3 & 1,02 & 0,31 & 1,37 & $>0,99 \mathrm{NS}$ & 0,29 & 1,11 & 0,28 & 1,31 & $>0,99 \mathrm{NS}$ \\
\hline$y$ Go & 0,4 & 1,24 & 0,35 & 1,14 & $>0,99 \mathrm{NS}$ & 0,39 & 1,28 & 0,34 & 1,26 & $>0,99 \mathrm{NS}$ \\
\hline$x \mathrm{Ar}$ & 0,09 & 0,65 & 0,12 & 0,83 & $>0,99 \mathrm{NS}$ & 0,09 & 0,69 & 0,09 & 0,53 & $>0,99 \mathrm{NS}$ \\
\hline$y \mathrm{Ar}$ & 0,08 & 0,48 & 0,12 & 0,66 & $>0,99 \mathrm{NS}$ & 0,1 & 0,61 & 0,09 & 0,58 & $>0,99 \mathrm{NS}$ \\
\hline$x \mathrm{Ba}$ & 0,35 & 1,86 & 0,25 & 1,37 & $>0,99 \mathrm{NS}$ & 0,29 & 1,93 & 0,2 & 0,88 & $>0,99 \mathrm{NS}$ \\
\hline$y \mathrm{Ba}$ & 0,26 & 1,28 & 0,27 & 1,5 & $>0,99 \mathrm{NS}$ & 0,22 & 1,02 & 0,22 & 0,9 & $>0,99 \mathrm{NS}$ \\
\hline$x$ Por & 0,21 & 1,73 & 0,3 & 1,84 & $>0,99 \mathrm{NS}$ & 0,12 & 0,7 & 0,21 & 1,29 & $>0,99 \mathrm{NS}$ \\
\hline$y$ Por & 0,1 & 0,68 & 0,12 & 0,6 & $>0,99 \mathrm{NS}$ & 0,13 & 1,09 & 0,14 & 0,69 & $>0,99 \mathrm{NS}$ \\
\hline$x \mathrm{Op}$ & 0,93 & 3,5 & 0,97 & 3,66 & $>0,99 \mathrm{NS}$ & 1,02 & 3,44 & 0,92 & 3,71 & $>0,99 \mathrm{NS}$ \\
\hline$y$ Op & 0,18 & 0,86 & 0,22 & 1,18 & $>0,99 \mathrm{NS}$ & 0,2 & 0,93 & 0,23 & 0,84 & $>0,99 \mathrm{NS}$ \\
\hline
\end{tabular}


Tableau 4

Moyenne des écart-types et des surfaces de dispersion des points.

\begin{tabular}{|c|c|c|c|c|c|c|c|c|c|c|}
\hline & $S$ & $\mathrm{Na}$ & Or & ENA & ENP & A & IS apex & IS bl & II bl & II apex \\
\hline$x \mathrm{SD}$ & 0,043 & 0,045 & 0,225 & 0,053 & 0,438 & 0,03 & 0,128 & 0,035 & 0,06 & 0,13 \\
\hline$y \mathrm{SD}$ & 0,053 & 0,07 & 0,145 & 0,048 & 0,06 & 0,095 & 0,13 & 0,048 & 0,053 & 0,185 \\
\hline \multirow[t]{2}{*}{$\mathrm{S}\left(\mathrm{mm}^{2}\right)$} & 0,701 & 0,99 & 10,25 & 0,783 & 8,247 & 0,895 & 5,207 & 0,522 & 0,99 & 7,556 \\
\hline & 0 & 0 & & o & $\longrightarrow$ & 0 & & 0 & 0 & \\
\hline Ordre & 2 & 6 & 16 & 3 & 15 & 5 & 12 & 1 & 6 & 13 \\
\hline
\end{tabular}

\begin{tabular}{|c|c|c|c|c|c|c|c|c|c|c|}
\hline & B & Pm & Pog & $\mathrm{Me}$ & Go & Ar & $\mathrm{Ba}$ & Por & Op & Moy \\
\hline$x \mathrm{SD}$ & 0,033 & 0,038 & 0,043 & 0,075 & 0,295 & 0,098 & 0,273 & 0,21 & 0,96 & 0,106 \\
\hline y SD & 0,148 & 0,2 & 0,178 & 0,038 & 0,37 & 0,098 & 0,243 & 0,123 & 0,208 & 0,084 \\
\hline $\mathrm{S}\left(\mathrm{mm}^{2}\right)$ & 1,506 & 2,356 & 2,37 & 0,884 & 34,290 & 2,986 & 20,76 & 8,082 & 65,58 & 2,821 \\
\hline & 0 & 0 & 0 & 0 & & $\bigcirc$ & & & & \\
\hline Ordre & 8 & 9 & 10 & 4 & 18 & 11 & 17 & 14 & 19 & \\
\hline
\end{tabular}

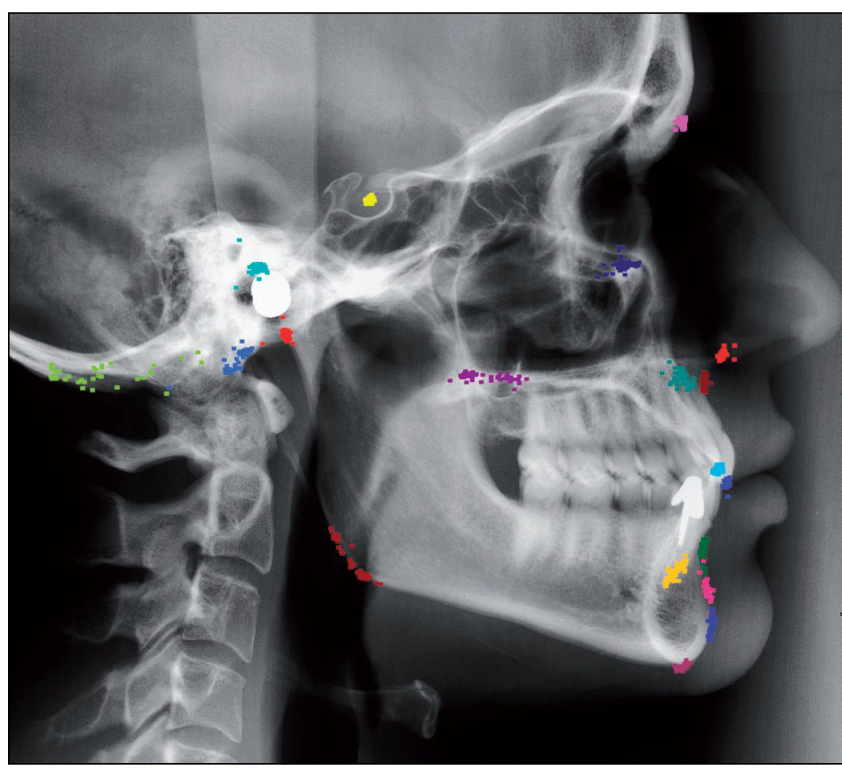

Figure 2

Dispersion réelle des points céphalométriques.
Aucune publication n'a été retrouvée proposant un échantillon proche de celui de cette étude. Le matériel et la méthode diffèrent par le nombre de participants (1 à 12), le nombre de clichés tracés (1 à 5), le plus souvent issus de sujets multiples. Aucune étude n'analyse à la fois l'influence de la distance et du récepteur, sur un seul et même sujet, par un aussi grand nombre de praticiens, ainsi que le relève également Leonardi notant l'absence d'études statistiquement significatives [19].

\subsection{Influence du récepteur}

Notre étude ne met pas en évidence de différence statistiquement significative liée au type de récepteur utilisé. Seuls Lim et Foong puis Geelen ont analysé l'influence du type de récepteur, en utilisant des films argentiques et des capteurs numériques au phosphore, sur la précision d'identification de points [13, 20]. Nos résultats viennent conforter ces deux études et soulignent une nouvelle fois l'intérêt des technologies d'imagerie numérique qui autorisent une diminution d'exposition significative. 
Tableau 5

Moyennes, écarts-types et classement des mesures angulaires et linéaires.

\begin{tabular}{|c|c|c|c|}
\hline Mesures angulaires & Moyenne & $\begin{array}{l}\text { Écart } \\
\text { type }\end{array}$ & Classement \\
\hline SNA & 80,73 & 0,63 & 5 \\
\hline SNB & 78,77 & 0,58 & 3 \\
\hline ANB & 1,96 & 0,27 & 1 \\
\hline FMIA & 67 & 2,25 & 17 \\
\hline IMPA & 94,79 & 2,02 & 12 \\
\hline FMA & 18,08 & 1,19 & 10 \\
\hline Angle inter-incisif & 134,75 & 2,95 & 20 \\
\hline Axe I / pl. Francfort & 112,34 & 2,82 & 19 \\
\hline Angle facial & 92,70 & 1,11 & 9 \\
\hline Angle de convexité & 2,99 & 0,62 & 4 \\
\hline (A-B) / pl. facial & $-3,60$ & 0,52 & 2 \\
\hline Axe Y / pl. Francfort & 53,58 & 0,92 & 8 \\
\hline$(\mathrm{S}-\mathrm{Na}) / \mathrm{pl}$. mand. & 27,54 & 2,22 & 15 \\
\hline Axe I / (Na-A) & 18,07 & 2,38 & 18 \\
\hline Axe i / (Na-A) & 27,02 & 1,96 & 11 \\
\hline Pl. max. / pl. mand. & 22 & 0,87 & 7 \\
\hline Ligne biapic. / pl. max. & 83,67 & 2,23 & 16 \\
\hline Pl. mand. / ligne biapic. & 74,35 & 2,14 & 14 \\
\hline Angle facial (Ricketts) & 92,65 & 0,63 & 5 \\
\hline I / (A-Pog) & 24,16 & 2,03 & 13 \\
\hline \multicolumn{4}{|l|}{ Mesures linéaires } \\
\hline Hauteur face & 12,43 & 0,07 & 3 \\
\hline Étage supérieur & 6,09 & 0,12 & 8 \\
\hline Étage inférieur & 6,34 & 0,10 & 6 \\
\hline Hauteur face post. & 5,46 & 0,46 & 11 \\
\hline Protrusion & 0,36 & 0,06 & 2 \\
\hline II Pt-A II & 6,84 & 0,12 & 8 \\
\hline II Pt-Gna II & 10,98 & 0,09 & 5 \\
\hline II Ar-A II & 9,64 & 0,11 & 7 \\
\hline II Ar-Gna II & 12,04 & 0,13 & 10 \\
\hline Diff. max.-mand. & 2,40 & 0,07 & 3 \\
\hline Dimension vert. face & 7,14 & 0,05 & 1 \\
\hline
\end{tabular}

\subsection{Influence de la distance}

L'analyse statistique des résultats n'a pas permis également de mettre en évidence de différence statistiquement significative entre les deux distances foyersujet présentées et ce pour chacun des 19 points céphalométriques analysés. Ces résultats sont à rapprocher de ceux de Hsiao, qui observe que des mesures effectuées sur crâne sec puis sur un cliché téléradiographique ne révèlent pas de déformations majeures (erreur inférieure à $0,5 \mathrm{~mm}$ après application d'un coefficient de réduction de 11,10) qui pourraient nuire à l'identification des points [17]. Selon Yoon et Leonardi, les points les plus affectés par les phénomènes de déformation liés à la projection de structures anatomiques en 3D sur un film en deux dimensions sont ceux les pluséloignés du foyer radiologique, donc les points les plus latéraux [19, 33]. Ce phénomène étant aggravé par la diminution de distance foyersujet, les points Articulare, Porion, Sous Orbitaire et Gonion devaient donc être les plus affectés à 1,50 m. Cette tendance, statistiquement non significative, est retrouvée dans les résultats de cette étude.

La moyenne des surfaces de dispersion des points Ar à $4 \mathrm{~m}$ est de $2,545 \mathrm{~mm}^{2}$, alors qu'elle est de $3,464 \mathrm{~mm}^{2}$ à $1,50 \mathrm{~m}$. Il en est de même pour le point Porion qui présente une moyenne de dispersion de $5,961 \mathrm{~mm}^{2}$ à $4 \mathrm{~m}$ contre $10,41 \mathrm{~mm}^{2}$ à $1,50 \mathrm{~m}$. Le conduit auditif, oblique par rapport au rayon incident, présente sur sa partie supérieure une zone de flou, plus marquée à 1,50 m, ce qui altère l'identification du point Po. Cette augmentation d'imprécision est également retrouvée pour le point Or $\left(5,812 \mathrm{~mm}^{2}\right.$ contre $15,82 \mathrm{~mm}^{2}$ ), alors que les 16 autres structures ne sont pas ou peu affectées.

Le point Gonion, pourtant bilatéral, présente une surface plus faible à $1,5 \mathrm{~m}$ qu'à $4 \mathrm{~m}$ : il reste cependant un des points présentant un nuage d'erreur le plus étendu (classé avant dernier devant Op en termes de surface de dispersion). Ceci peut être expliqué par sa situation sur une convexité osseuse à faible progression mais aussi par la superposition avec l'angle goniaque controlatéral.

Vincent et West, dans une étude sur la fiabilité portant sur 22 points, classent également, parmi les 13 points communs à notre étude, Gonion en dernière position [31].

\subsection{Fiabilité des points céphalométriques}

Les différents types de dispersion observés sont rapportés dans plusieurs études ; Baumrind et Frantz 
ont été les premiers en 1971 à proposer un classement des points en fonction de leur fiabilité $[4,5]$. Aux premières places figurent, dans l'ordre : le bord libre incisif supérieur, le bord libre incisif inférieur, $\mathrm{S}, \mathrm{Na}, \mathrm{Me}$, A, Pog (ENA n'étant pas analysé).

De même, Broch obtient un classement similaire, à l'exception de ENA qui se retrouve mieux classé que Pog, lui-même mieux classé que le point A [9]. Seule Geelen, analysant les points sur clichés argentiques et numériques, place le point Articulare parmi les 5 points les plus fiables $(\mathrm{SD}<0,75 \mathrm{~mm}$ ) devant ENA et $\mathrm{Me}(0,75<\mathrm{SD}<1,5 \mathrm{~mm})$ [13]. Vincent et West, quant à eux, obtiennent comme points les plus fiables: Me, bord libre incisive supérieure, S, A [31].

Globalement nos résultats mettent en évidence que les points situés sur l'axe médian et dans les zones de haut contraste, donc souffrant moins souvent de la superposition de structures anatomiques, sont les plus fiables, confirmant les constatations de plusieurs auteurs $[4,9,18,24,29,31]$. Ainsi les points médians squelettiques ( $, \mathrm{Na}, \mathrm{ENA}, \mathrm{Me}$ ) et dentaires (bords libres incisifs maxillaire et mandibulaire) ont des nuages de dispersion caractéristiques, de forme circulaire et peu étendue. Ces points peuvent donc être considérés comme les plus fiables : notre étude classe, tous type de clichés confondus, le bord libre de l'incisive supérieure en première position, suivi par S, ENA, $\mathrm{Me}, \mathrm{A}$ puis $\mathrm{Na}$ et bord libre de l'incisive inférieure à égalité.

La grande stabilité du point S peut être expliquée par la facilité par l'œil de localiser le centre d'une structure circulaire. La très légère dispersion sur l'axe vertical est probablement justifiée par l'ouverture de la selle turcique sur son bord supérieur.

Le point Na présente un nuage de dispersion également circulaire, mais légèrement plus dispersé : cette observation peut s'expliquer par une hésitation, lors de son identification, liée à la suture naso-frontale elle-même. Théoriquement placée sur la berge supérieure, de nombreux participants ont localisé ce point, malgré le rappel de sa définition, sur sa berge inférieure.

Un plan tel que S-Na pourrait ainsi, et comme certains auteurs l'ont déjà proposé, remplacer avantageusement le plan de Francfort [4, 23]. Les points Po et Or, permettant la construction de ce dernier, ont en effet tous les deux des nuages de dispersions les classant parmi les repères « peu fiables » (respectivement $14^{\mathrm{e}}$ et $16^{\mathrm{e}}$ ). Le point Po présente une dispersion principalement sur l'axe des $X$, suivant le conduit du méat acoustique externe, le point Or présente une disper- sion similaire suivant le contour inférieur de l'orbite. Une critique du plan de Francfort (décrit en 1884), le plus utilisé pour l'orientation de la tête, semble donc permise, comme l'observaient déjà dans leurs études respectives Pancherz, Foster et Richardson [12, 23, 24]

Le bord libre de l'incisive supérieure présente également une grande stabilité, puisqu'il présente le nuage de dispersion le plus réduit $\left(0,522 \mathrm{~mm}^{2}\right.$ tous types de clichés confondus), lui conférant la première position du classement. Le bord libre de l'incisive inférieure est déjà beaucoup moins précis, puisque classé en $6^{e}$ position. Ce résultat s'explique probablement par la superposition de l'ensemble des dents antérieures et de leurs bords libres, répartis sur un arc de cercle de plus faible amplitude. Les apex de l'incisive maxillaire et surtout mandibulaire sont nettement moins regroupés que les bords libres. Staburn constatait déjà que dans $75 \%$ des cas l'apex de l'incisive inférieure était mal localisé [29]. Il semblerait que les praticiens, lorsqu'ils ne visualisent pas directement l'apex, utilisent leurs connaissances de l'anatomie dentaire pour estimer la longueur de la dent, la comparant à celles des dents adjacentes. Suivre les axes de convergence des bords de la racine peut également permettre de localiser l'apex. Mais ces deux méthodes de « déduction » peuvent être d'autant plus sévèrement perturbées que l'encombrement incisivo-canin est important, ce phénomène étant généralement plus marqué à la mandibule.

Le point ENA qui, d'après Hägg, est plus ou moins identifiable selon l'anatomie du sujet radiographié et l'épaisseur des tissus mous [14], est bien visible sur les quatre clichés de l'étude ; son nuage de dispersion est circulaire et bien groupé. Le nuage apparait toutefois plus étendu sur le cliché numérique à $4 \mathrm{~m}$. Ce résultat peut s'expliquer par le contraste général de ce cliché plus élevé, qui renforce la radio-opacité des ailes du nez, superposées anatomiquement à ENA.

Les points situés le long des corticales osseuses nettes présentent une grande stabilité, sous réserve de ne pas être situées tangentiellement à une structure présentant une concavité ou une convexité peu marquée. Baumrind et Frantz, Staburn, Vincent et Hägg rapportaient déjà ce phénomène $[4,14,29,31]$. Ainsi les relevés des points A, B, Pog, Pm présentent dans notre étude des nuages de dispersion stables sur l'axe horizontal, mais peu sur l'axe vertical : la forme du nuage épouse celle de la structure anatomique, autant pour l'os alvéolaire que la symphyse mandibulaire. L'étude de Hägg réalisée sur crânes secs présentait 
graphiquement les points portés par la face antérieure de la symphyse mandibulaire et obtenait le même type d'« étalement» sur l'axe des Y [14]. Ces quatre points pourront donc être considérés comme fiables pour réaliser des mesures antéro-postérieures, du fait de leur stabilité sur l'axe des $X$, mais devront être utilisés avec précaution pour des mesures verticales.

Le point Ophistion présente une dispersion anarchique sur l'axe des $X$, moins sur l'axe des $Y$; ces résultats sont explicables par la méconnaissance du point, ainsi que par sa très faible utilisation dans les différentes analyses céphalométriques. Malgré sa définition précise fournie, les connaissances anatomiques d'une très grande majorité des observateurs n'ont pas été suffisantes pour parvenir à déduire la localisation de ce point. Peu d'entre eux avaient le souvenir que le foramen magnum, délimité antérieurement par Ba et postérieurement par Op, présente un diamètre moyen de 3,5 cm, absolument nécessaire au passage de la moelle épinière. Cette distance a été sous évaluée dans la quasi totalité des tracés. Le point Ba, situé dans une zone de faible contraste lié à la superposition des structures, n'est également pas localisé avec une grande précision. De plus, la majorité des observateurs ont déclaré utiliser l'analyse de Tweed, celle-ci n'employant pas ce repère souvent difficile à situer. Son nuage de dispersion s'étale plutôt sur l'axe des $X$, la longueur basi-occipitale n'étant pas toujours aisément mesurable. Ces résultats sont similaires à ceux observés par Arponen [3].

\subsection{Reproductibilité des mesures}

À l'exception de Kamoen, la littérature ne rapporte pas de classement des mesures angulaires et linéaires similaires, la majorité des études analysant 2 à 5 angles issus des analyses céphalométriques [18]. Néanmoins, les classements "partiels » proposés par Houston, Cook et Gravely sont compatibles tant avec les résultats observés dans cette étude qu'avec ceux de Kamoen $[11,16]$.

Les mesures les plus fiables sont ainsi liées à l'analyse du sens antéro-postérieur qui traduit le décalage des bases osseuses (ANB, AB/plan facial, SNB, angle de convexité, SNA).

Les mesures angulaires qui présentent les plus forts écarts-types $\left(>2^{\circ}\right)$, traduisant les plus faibles précisions, sont, à l'exception de l'angle S-NA / plan mandibulaire, toutes liées à la position des apex des incisives maxillaires ou mandibulaires: angle inter-incisif, axe incisif supérieur / plan de Francfort, axe incisif supérieur / Na-A, plan maxillaire / ligne bi-apicale, plan mandibulaire / ligne bi-apicale, axe incisif inférieur / A-Pog, IMPA et axe incisif inférieur / Na-A. Ces observations rejoignent celles de Staburn [29] et conduisent à s'interroger sur cette référence céphalométrique largement utilisée, le redressement de l'incisive mandibulaire étant l'un des objectifs reconnus des traitements orthodontiques.

La variation de l'angle S-NA / plan mandibulaire précédemment évoquée s'explique par l'utilisation du point Go (analyse de Steiner) : l'utilisation du point de tangence inférieur de la mandibule apparaît plus fiable lors de l'évaluation du plan mandibulaire.

À l'exception de la hauteur faciale postérieure, qui utilise une nouvelle fois le point Go (analyse de TweedMerrifield), toutes les mesures linéaires présentent une variabilité similaire et inférieure à $0,11 \mathrm{~mm}$.

L'analyse des erreurs moyennes des mesures angulaires et linéaires rapportées à chaque analyse céphalométrique montre que les erreurs sont respectivement comprises entre $1,26^{\circ}$ et $1,63^{\circ}$ et entre $0,18 \mathrm{~mm}$ et $0,36 \mathrm{~mm}$. Aucune des analyses testées ne montre donc une susceptibilité particulière, ces résultats étant comparables avec ceux de Kamoen [18].

\section{Conclusion}

Seule étude réalisée sur un grand nombre de praticiens aléatoirement sélectionnés, les résultats issus de son analyse montrent qu'il n'existe aucune différence statistiquement significative liée à la distance foyer-sujet ou au type de récepteur (argentique / numérique) lors de l'identification des repères céphalométriques. Le type de cliché qui présente en moyenne les nuages de dispersion les plus faibles est celui réalisé sur une installation à 1,50 m associé à un capteur numérique. Ces résultats confirment la fiabilité de ce type d'installation, dont les chiffres de vente en unité augmentent régulièrement depuis ces dernières années, supplantant les installations argentiques de téléradiographie à $4 \mathrm{~m}$. La numérisation autorise donc une diminution de dose d'irradiation compatible avec le maintien de la puissance diagnostique.

Chaque point céphalométrique présente un nuage caractéristique de dispersion.

Les points situés sur l'axe sagittal médian et dans une zone de haut contraste radiologique sont les plus fiables, tandis que les points bilatéraux ou situés dans une zone présentant d'importantes superpositions anatomiques sont les moins fiables. 
Les points localisés sur des structures anatomiques dont la forme présente une concavité ou une convexité à faible progression ont des nuages de dispersion qui épousent le contour de celles-ci.

Consécutivement aux erreurs de localisations des points céphalométriques, les erreurs de mesures angulaires et linéaires sont faibles (respectivement inférieures à $3,1^{\circ}$ et $0,46 \mathrm{~mm}$ ). Néanmoins, celles qui présentent les variations les plus importantes sont celles liées à l'identification des apex incisifs, ce qui peut légitimement conduire à relativiser l'apport diagnostic et thérapeutique de l'analyse des positions incisives lors des traitements orthodontiques. Cette analyse peut également être appliquée au plan de Francfort qui apparaît être un plan de référence moins fiable que S-Na.

\section{Bibliographie}

[1] AdenwallaST, KronmanJH, AttarzadehF. Porionand condyle as cephalometric landmarks - An error Study. Am J Orthod Orthofacial Orthop 1988;94:411-415.

[2] Ahlqvist J, Eliasson S, Welander U. The effect of projection errors on angular measurements in cephalometry. Eur J Orthod 1988;10:353-361

[3] Arponen H, Elf H, Evalahti M, Waltimo-Siren J. Reliability of cranial base measurements on lateral skull radiographs. Orthod Craniofac Res 2008;11:201-210.

[4] Baumrind S, Frantz R. The reliability of head film measurements: 1, Landmark identification. Am J Orthod 1971;60:111-127.

[5] Baumrind S, Frantz R. The reliability of head film measurements: 2, Conventional angular and linear measurements. Am J Orthod 1971;60:505-517.

[6] Björk A. The face in profile. An anthropological X-ray investigation on Swedish children and conscripts. Svenska Tandlakare-Tidskrist 1947;40:5B

[7] Bourriau J, Cabot I, Foucart JM. Téléradiographie. Encyclopédie Médico Chirurgicale, Odonto/Stomatologie, Paris: Elsevier ; 1999, 23-455-E-10, 9 p.

[8] Broadbent BH. A new X-ray technique and its applications to orthodontics. Angle Orthodontist 1931;1:45-66.

[9] Broch J, Slagsvold O, Rosler M. Error in landmark identification in lateral radiograph head plates. Eur J Orthod 1981;3:9-13

[10] Cohen AM. Uncertainty in cephalometrics. Br J Orthod 1984;11:44-48.

[11] Cook PA, Gravely JF. Tracing errors with Björk's mandibular structures. Angle Orthod 1988;58:169-178.

[12] Foster TD, Howat AP, Naish PJ. Variation in cephalometric reference lines. Br J Orthod 1981;8:183-187.

[13] Geelen W, Wenzel A, Gotfredsen E, Kruger M, Hansson LG. Reproductibilty of cephalometric landmarks on conventional film, hardcopy, and monitor-displayed images obtained by the storagephosphore technique. Eur J Orthod 1998:20:331-340
[14] Hägg U, Cooke MS, Chan TC, Tng TT, Lau PY. The reproductibility of cephalometric landmarks : an experimental study on skulls. Aust Orthod J 1998;15:177-185.

[15] Haynes S, Chau MN. Inter- and intra-observer identification of landmarks used in the Delaire analysis. Eur J Orthod 1993;15:79-84.

[16] Houston WJB. The analysis of errors in orthodontic measurements. Am J Orthod 1983;83:382-390.

[17] Hsiao TH, Chang HP, Liu KM. A method of magnification correction for posteroanterior radiographic cephalometry. Angle Orthod 1997;67:137-142

[18] Kamoen A, Dermaut L, Verbeeck R. The clinical significance of error measurement in the interpretation of treatment results. Eur J Orthod 2001;23:569-578.

[19] Leonardi R, Annunziata A, Caltabiano M. Landmark identification error in posteroanterior cephalometric radiography cephalometric radiography. Angle Orthod 2008;78:761765

[20] Lim KF, Foong KWC. Phosphor-stimulated computed cephalometry: reliability of landmark identification. Br J Orthod 1997:24:301-308

[21] Macri V, Wenzel A. Reliability of landmark recording on film and digital lateral cephalograms. Eur J Orthod 1993;15:137148

[22] Midtgard J, Bjork A, Linder-Aronson S. Reproductibility of cephalometric landmarks and errors of measurements of cephalometric cranial distances. Angle Orthod 1974;44:56-61

[23] Pancherz H, Gökbuget K. The reliability of the Franckfor horizontal in roentgenographic cephalometry. Eur J Orthod 1996;18:367-372.

[24] Richardson A. An investigation into the reproductibility of some points, planes and lines used in cephalometric analysis Am J Orthod 1966;52:637-651

[25] Savage AW, Showfety KJ, Yancey J. Reapeted measures analysis of geometrically constructed and directly determined cephalometric points. Am J Orthod Dentofacial Orthop 1987:91:295-299.

[26] Savara RS, Tracey WE, Miller PA. Analysis of error in cephalometrics measurements of three dimensional distances on the human mandibule. Arch Oral Biol 1966;11:209-217.

[27] Seki K, Okano T. Exposure reduction in cephalometry with a digital photostimulable phosphor imaging system. Dentomaxillofac Radiol 1993;22:127-130.

[28] Spolyar JL. Head positioning error in cephalometric radiology. Angle Orthod 1987;57:77-88.

[29] Stabrun AE, Danielsen K. Precision in cephalometric landmark identification. Eur J Orthod 1982;4:185-196

[30] Trpkova B, Major P, Prasad N, Nebbe B. Cephalometric landmarks identification and reproductibility: a meta analysis. Am J Orthod Dentofacial Orthop 1997;112:165-170.

[31] Vincent AM., West VC. Cephalometric landmark identification error. Aust Orthod J 1987;10:98-104.

[32] Wah PY, Cooke MS, Hagg U. Effects of training and experience on cephalometric measurement errors on surgical patients. Int J Adult Orthodont Orthognat Surg 1997;12:204-213.

[33] Yoon YJ, Kim DH, Yu PS, Kim HJ, Choi EH, Kim KW. Effect of head rotation on posteroanterior cephalometric radiographs. Angle Orthod 2002;72:36-42. 